\title{
Comparison of Recurrence of Non-muscle Invasive Bladder Carcinoma after Transurethral Resection with Hexaminolevulinate Photodynamic Diagnosis or Regular Cystoscopy
}

Fokke Hoogeveen ( $\nabla$ sebastiaanhoogeveen@gmail.com ) Isala

Marco Blanker

University Medical Center Groningen

Evelyne Cauberg

Isala

Martijn Steffens

Isala

Research Article

Keywords: hexaminolevulinate, photodynamic diagnosis (PDD), white light cystoscopy (WLC), carcinoma

Posted Date: January 6th, 2021

DOI: https://doi.org/10.21203/rs.3.rs-138817/v1

License: (1) This work is licensed under a Creative Commons Attribution 4.0 International License. Read Full License 


\section{Abstract}

Purpose: Comparison of recurrence of non-muscle invasive bladder carcinoma after transurethral resection with hexaminolevulinate photodynamic diagnosis (PDD) or regular white light cystoscopy (WLC).

Methods: We included patients with newly suspected non-muscle invasive bladder carcinoma in this retrospective cohort study and compared those undergoing transurethral resection by WLC and PDD. The primary outcome was the difference in the recurrence rate after 60 months' follow-up, but we also stratified recurrence by risk groups. The mean recurrence-free survival was compared between the cohorts. Odds ratios or hazard ratios are reported with $95 \%$ confidence intervals.

Results: The WLC and PDD cohorts comprised 124 and 91 subjects, respectively. There were no significant differences in recurrence rates at 6 months (recurrence rate 9/123; 7.3\%), 12 months (17/118; 14.4\%), or 60 months (39/102; 38.2\%), with odds ratios of $1.23(0.48-3.25 ; P=0.64), 1.32(0.67-2.62 ; P=0.42)$, and 1.12 $(0.70-1.79 ; P=0.65)$, respectively. Further analysis showed no significant effect of PDD on recurrence by risk group or on mean recurrence-free survival (hazard ratio, 1.12; 0.70-1.79).

Conclusion: Photodynamic diagnosis with hexaminolevulinate did not reduce the recurrence of non-muscle invasive bladder carcinoma compared to standard white light cystoscopy when used for transurethral resection.

\section{Introduction}

Non-muscle invasive bladder cancer (NMIBC) accounts for most bladder cancers and can usually be treated by transurethral resection (TUR)[1]. However, the efficacy of TUR is highly variable, with recurrence rates (RR) of up to $78 \%$ at 5 years[2]. Early recurrence results from incomplete resection of the primary tumor or the presence of undetected tumors[3]. The current standard approach for TUR involves white light cystoscopy (WLC), which is effective for detecting papillary tumors. However, carcinoma in situ (CIS), small satellite growths, or dysplasia can be missed[4].

To improve tumor detection, photodynamic diagnosis (PDD) was developed that uses photoactive porphyrins that accumulate in neoplastic tissue and become fluorescent under blue light [4]. Hexaminolevulinate (Hexvix ${ }^{\mathrm{T}}$; Ipsen, France) is one such photoactive agent that has been shown to increase tumor detection rates by $9.7-40.2 \%[5,6]$. The effect of PDD on RR has therefore gained interest, despite systematic reviews and meta-analyses of randomized controlled trials showing varied results. In 2012, Shen reported no effect on short-term recurrence in a systematic review of different fluorescent agents, including hexaminolevulinate[7]. Shortly thereafter, Yuan reported a significant effect of PDD on the time to first recurrence and on the recurrence-free survival (RFS) at 1 and 2 years[8], whereas Burger reported an insignificant effect on time to recurrence and a significant effect on the RR for hexaminolevulinate alone[6]. A meta-analysis by Chou in 2017 showed that hexaminolevulinate use had a significant effect on recurrence[9].

Most reviews published have reported the quality of evidence to be low, mainly because of heterogeneity (e.g., trials included both new and recurrent carcinoma), lack of treatment standardization (e.g., intravesical chemotherapy), and lack of subgroup analysis[7-9]. Nevertheless, despite the inconclusive reports, PDD has 
been introduced in clinical care. In a prospective single-center study, Gallagher showed that using PDD routinely in a real-world setting significantly decreased the long-term recurrence of NMIBC. In their research, treatment was standardized with recommended "good quality resection" and the instillation of postoperative mitomycin $C(M M C)[10,11]$. Among their 354 subjects, the RR after 3 years of follow-up $\square$ decreased from $53.3-39.0 \%$, giving a relative risk reduction of $56 \%$ and a $95 \%$ confidence interval $(95 \% \mathrm{Cl})$ of $0.50-0.94$. We are unaware of more recent studies supporting these outcomes in real-world settings.

In our hospital, PDD replaced WLC in 2010 as the standard method for TUR when treating patients with suspected NMIBC. We therefore sought to confirm the results of Gallagher[11] in our population. In this study, we compared how hexaminolevulinate-based PDD and WLC TUR affected the recurrence of newly presenting NMIBC over a 5-year follow-up period.

\section{Materials And Methods}

\section{Study Design}

We conducted a retrospective cohort study in our hospital, covering a period that spanned from before to after the introduction of PDD in 2010. Before the introduction of PDD all patients suspected of bladder carcinoma underwent TUR with WLC. Afterwards, only patients with newly suspected NMIBC underwent a standardized TUR procedure with PDD whereas patients with suspected muscle invasive carcinoma were excluded.

We compared the outcomes of consecutive patients newly presenting with NMIBC and treated in our hospital between January, 2008, and December, 2012. This resulted in time series for a WLC cohort (from 2008 to PDD introduction) and a PDD cohort (from PDD introduction onward) that were mutually exclusive. This study duration allowed for a follow-up period of 5 years in all cases. The primary outcome was the time to recurrence. Follow-up was performed by WLC in both groups, according to European Association of Urology (EAU) guidelines[12].

\section{Patient Selection}

Eligible patients were identified from our patient directory based on the operation performed, using the $\mathrm{CTcue}^{\mathrm{TM}}$ software (CTcue, the Netherlands). Patients were excluded if the resection did not meet the good quality resection criteria, PDD was unsuccessful (absence of fluorescent signal), muscle invasive carcinoma was suspected at first cystoscopy or pathologically proven after TUR, malignancy other than urothelial cell carcinoma (UCC) was found, or if patients did not undergo adjuvant intravesical chemotherapy or immunotherapy if indicated.

\section{Procedures}

To standardize resection quality, we applied the principles of good quality resection, as suggested by Mariappan, with the exception of cystoscopic bladder mapping which was not reported in the retrospective data[10]. We applied the following principles[10]: resections carried out by a urologist, or by a urology resident under close supervision; all visible and otherwise documented tumors resected; Detrusor muscle resected either en masse or by fractionated resections; and, postoperative intravesical MMC instilled. Cystoscopy and bipolar resection were performed in both the PDD and WLC cohorts using a 27 French gage Olympus 
resectoscope (type A22041). According to the manufacturer's guidelines, we instilled $50 \mathrm{~mL}$ of phosphate buffered saline containing $85 \mathrm{mg}$ of hexaminolevulinate through the catheter, retained it for 60 minutes, and drained it prior to cystoscopy[13].

\section{Data collection}

We retrieved the following data from the medical files of included patients: age at first treatment, histological analysis (e.g., tumor stage, tumor grade, presence of $\mathrm{CIS}$ ), single postoperative MMC instillation, adjuvant intravesical chemotherapy or immunotherapy, date of first recurrence, and date and cause of death. Patient survival was based on data from the Municipal Population Register. Malignancies were divided by European Organization for Research and Treatment of Cancer (EORTC) risk group, with the exception of tumor size, which was not systematically recorded in our data[2]. Ethical approval and informed consent collection were waived by the local Ethics Committee of the Isala Hospital in Zwolle, the Netherlands, in view of the retrospective nature of the study and all the procedures being performed were part of routine care.

\section{Statistical analysis}

Analyses were performed using IBM SPSS Version 25 (IBM Corp, USA). We considered a P-value of $\leq 0.05$ statistically significant. Descriptive analyses were performed for the baseline characteristics based on their distributions, and differences between the two cohorts were tested using the chi-square test and t-test. The cumulative overall death rate, as well as the number of deaths due to confirmed UCC progression, were compared between study groups using the chi-square test.

The primary outcome, time until first recurrence, was compared between the two groups over the 5-year follow-up period using Cox proportional logistic regression. Study group was entered as the explanatory variable, and censoring was defined as loss to follow-up or death. We checked for imbalanced censoring between the groups by chi-square test of the percentage of censored patients. The resulting data are presented in survival curves with accompanying hazard ratios. Univariate Cox regression analyses were then performed to adjust for the following possible confounders: tumor stage, tumor grade, CIS presence, EORTC risk group, and receipt of adjuvant intravesical therapy. Confounders were chosen for their putative influence on recurrence according to the EAU guideline on NMIBC[12]. Propensity score analysis was performed to adjust for these confounders, using univariable Cox regression analyses with treatment group as the dependent variable and characteristics with a significant imbalance between both groups as independent variables. Any variable with a univariate P-value of $\leq 0.25$ was entered into the multivariate logistic regression analysis to calculate the propensity score. Finally, the resulting propensity score was entered in a multivariate Cox regression analysis, using RFS as the dependent variable.

\section{Results}

We identified 408 patients who underwent TUR during the study period, of these $215(52.7 \%)$ met the inclusion criteria (Fig. 1). The baseline characteristics are summarized in Table 1, more T1 tumors were present in the WLC group than the PDD group $(P=0.006)$ and there was a difference in adjuvant intravesical therapy use between the groups $(P=0.01)$. 
In total, 137 patients completed the 5-year follow-up. The number of deaths was considerably higher in the WLC group $(n=54 ; 43.5 \%)$ than in the PDD group $(n=24 ; 26.4 \%, P=0.01)$, but the number of deaths by recurrence was comparable. 38 patients $(70 \%)$ died recurrence free and $16(30 \%)$ died after recurrence in the WLC group, whereas 17 (70.8\%) died recurrence free and 7 (29.2\%) died after recurrence in the PDD group (P $=0.97$ ). Confirmed UCC progression was present in $14.1 \%$ of deaths, and there were more UCC-related deaths in the WLC group $(16.7 \%)$ than in the PDD group $(8.3 \% ; \mathrm{P}=0.33)$.

Table 1

Baseline characteristics

\begin{tabular}{|c|c|c|c|}
\hline & WLC & PDD & P value $^{*}$ \\
\hline \multicolumn{4}{|l|}{ Demographics } \\
\hline Number of patients & 124 & 91 & \\
\hline Median age (25-75 percentile) & $73(64-79)$ & $71.0(66-79)$ & 0.48 \\
\hline $\operatorname{Sex}(M / F)$ & $97 / 27$ & $72 / 19$ & 0.8 \\
\hline \multicolumn{4}{|l|}{ Tumor characteristics } \\
\hline T1 & $27(21.8)$ & $6(6.6)$ & 0.006 \\
\hline $\mathrm{CIS}$ & $11(8.9)$ & $8(8.8)$ & 0.98 \\
\hline Solitary & $81(65.3)$ & $61(67.1)$ & 0.59 \\
\hline Multifocal (>2) & $43(34.7)$ & $30(32.9)$ & \\
\hline \multicolumn{4}{|l|}{ Risk group } \\
\hline Low (TaG1, solitary, no CIS) & $32(25.8)$ & $27(29.7)$ & 0.52 \\
\hline Intermediate (TaG2, multifocal < 7, no CIS) & $46(37.1)$ & $37(40.6)$ & \\
\hline High (T1, G3, CIS, TaG1/G2 + > 7 tumors) & $46(37.1)$ & $27(29.7)$ & \\
\hline \multicolumn{4}{|l|}{ Adjuvant intravesical therapy } \\
\hline None or incomplete schedule & $60(48.4)$ & $54(59.3)$ & 0.01 \\
\hline BCG instillations & $20(16.1)$ & 15 (16.5) & \\
\hline MMC instillations & $44(35.5)$ & $22(24.2)$ & \\
\hline
\end{tabular}

RR at 6 months (7.3\%; 9/123), 12 months (14.4\%; 17/118), and 60 months (38.2\%; 39/102) were generally lower in the WLC group than in the PDD group (Table 2), with corresponding odds ratios of $1.23(95 \% \mathrm{Cl}, 0.48-$ $3.25 ; \mathrm{P}=0.64), 1.32(95 \% \mathrm{Cl}, 0.67-2.62 ; \mathrm{P}=0.42)$, and $1.12(95 \% \mathrm{Cl}, 0.70-1.79 ; \mathrm{P}=0.65)$, respectively. $\mathrm{RR}$ at 6 , 12 , and 60 months did not differ significantly by EORTC risk group. There was no significant difference between the groups in mean RFS, with a hazard ratio of 1.12 (95\%Cl,0.70-1.79; Fig. 2). 
Table 2

Recurrence rates within the study population, excluding censored cases
6 months
12 months
60 months

n (\%)

$\begin{array}{lll}\text { OR } & p & n(\%)\end{array}$

(\%) $\quad$ OR

OR
$(95 \% \mathrm{Cl})$

$\begin{array}{llll}\mathrm{p} & \mathrm{n}(\%) & \text { OR } & \mathrm{P} \\ \text { value }^{*} & & (95 \% \mathrm{Cl}) & \text { value }^{*}\end{array}$

All

\begin{tabular}{|c|c|c|c|c|c|c|c|c|}
\hline WLC & $\begin{array}{l}9 / 123 \\
(7.3)\end{array}$ & $\begin{array}{l}1.23 \\
(0.48- \\
3.25)\end{array}$ & 0.64 & $\begin{array}{l}17 / 118 \\
(14.4)\end{array}$ & $\begin{array}{l}1.32 \\
(0.67- \\
2.62)\end{array}$ & 0.42 & $\begin{array}{l}39 / 102 \\
(38.2)\end{array}$ & $\begin{array}{l}1.12 \\
(0.70- \\
1.79)\end{array}$ \\
\hline PDD & $\begin{array}{l}8 / 90 \\
(8.8)\end{array}$ & & & $\begin{array}{l}16 / 88 \\
(18.2)\end{array}$ & & & $\begin{array}{l}31 / 78 \\
(39.7)\end{array}$ & \\
\hline
\end{tabular}

\section{EORTC risk \\ group}

Low

WLC

$\begin{array}{lllll}4 / 32 & 0.02 & 0.06 & 5 / 30 & 0.22 \\ (12.5) & (0.00- & & (16.7) & (0.03- \\ & 54.5) & & & 1.87)\end{array}$

0.13

$8 / 23$

(34.8)

0.89

$(0.32-$

0.83

.87)

2.46)

PDD

$0 / 27$

$1 / 27$

(3.7)

$7 / 24$

(29.2)

Intermediate

WLC

$\begin{array}{lllll}1 / 46 & 3.89 & 0.20 & 5 / 44 & 2.15 \\ (2.2) & (0.40- & & (11.4) & (0.70- \\ & 37.40) & & & 6.57)\end{array}$

0.17

$11 / 34$

(32.4)

2.03

(0.93-

0.069

$37.40)$

$8 / 35$

(22.9)

$15 / 31$

(48.4)

(8.1)

High

WLC

$4 / 45$

(8.9)

2.33

(0.63-

8.69)

0.19

$7 / 43$

(16.3)

1.90

(0.67-

0.22

5.42)

$20 / 39$
$(51.3)$

0.78

(0.36-

0.58

PDD

$5 / 26$

(19.2)

$7 / 26$

(26.9)

$9 / 23$

(39.1)

*Log-rank test. Abbreviations: $\mathrm{Cl}$, confidence interval; EORTC, European Organization for Research and Treatment of Cancer; OR, odds ratio; PDD, photodynamic diagnosis; WLC, white light cystoscopy

Univariate Cox regression revealed that the number of lesions, the tumor stage, the tumor grade, the presence of $\mathrm{CIS}$, and the EORTC risk group were all possible confounders $(P<0.25)$, but adjuvant intravesical chemotherapy was not (Table 3). The Cox regression analyses adjusted for the propensity score, including these characteristics, indicated no significant difference between the WLC and PDD groups (hazard ratio,1.48; $95 \% \mathrm{Cl}, 0.89-2.48)$. 
Table 3

Identification of potential confounders

\begin{tabular}{|llll|}
\hline & Hazard Ratio* & $\mathbf{9 5 . 0 \%} \mathrm{Cl}$ & P value \\
\hline Number of lesions & 1.42 & $0.98-2.07$ & 0.064 \\
\hline T-Stage & 2.52 & $1.67-3.81$ & $>0.001$ \\
\hline T-Grade & 1.02 & $1.01-1.03$ & $>0.001$ \\
\hline Carcinoma in situ & 2.33 & $1.19-4.56$ & 0.013 \\
\hline EORTC risk group & 1.41 & $1.03-1.92$ & 0.031 \\
\hline Adjuvant intravesical therapy & 1.1 & $0.82-1.48$ & 0.51 \\
\hline *Cox regression analysis. Abbreviations: Cl, confidence interval; EORTC, \\
\hline \multirow{4}{*}{ European Organization for Research and Treatment of Cancer } \\
\hline
\end{tabular}

\section{Discussion}

This retrospective cohort study contributes to the small amount of real-world experience with PDD for TUR. Given that Gallagher published results that showed promise in a real-world setting[11], we set out to validate their outcomes using strict quality criteria to compare resections under optimal conditions in a retrospective design. However, we failed to show any significant difference in RR or mean RFS for NMIBC between the two groups. The question therefore arises as to how these results can be explained since they conflict with the trend seen in systematic reviews. The outcomes of NMBIC vary significantly based on a multitude of factors, making it difficult to investigate the role of any one factor on disease recurrence.

RR reported in the literature tend to be higher than in this study. The review by Di Stasi reported risk reductions of $14.8 \%$ and $32.3 \%$ at 12 months for PDD and WLC, respectively[5]. In the meta-analysis by Burger, corresponding RR of $₫ 34.5 \%$ and $45.4 \%$ at 12 months were reported $(P=0.006)$, whereas we showed rates of $18.2 \%$ and $14.4 \%$, respectively $(P=0.42)[6]$. However, it should be noted that most previous reviews have report populations in which both primary and recurrent bladder cancer were included. Given that patients with a history of recurrent disease will be at higher risk of future recurrence, one might expect a higher RR in these studies. Therefore, we chose to include only newly presenting cases of NMIBC, consistent with the reports of O'Brien and Gallagher. RR varied in those studies, which considered primary bladder cancer treated with a single dose of intravesical MMC after TUR. At 12 months, O'Brien reported rates of $16 \%$ for PDD and $22 \%$ for WLC $(P=0.4)$, whereas Gallagher reported slightly higher rates of $21.5 \%$ and $38.9 \%$, respectively $(P=0.02)[11$, 14]. This suggests that our PDD cohort does not necessarily perform worse, and that the WLC cohort performs slightly better compared to similar cohorts. 
In this study, the low and comparable RR between the PDD and WLC groups may be attributed to several factors. First, all resections were performed by experienced surgeons or by residents under close supervision. Second, not only did we adhere strictly to the EAU guidelines concerning adjuvant intravesical chemotherapy or immunotherapy we also used an instillation of postoperative MMC.

At baseline, our WLC group had more T1 tumors (21.8\%) than our PDD group (6.6\%). This led to a greater percentage of cases in the high-risk EORTC category in the WLC group (37.1\%) than in the PDD group (29.7\%). Although one might expect such high-risk tumors to recur more often than low- or intermediate-risk tumors, there was no significant difference in RR between the study groups. It should also be noted that the higher number of T1 tumors in the WLC group may have resulted from selection bias in the PDD group. For example, if patients were suspected of a muscle invasive bladder tumor at initial cystoscopy, they were selected to undergo WLC for TUR and therefore excluded from the study. Given that T1 tumors are more likely to appear macroscopically solid at cystoscopy, they are less likely to have been included in the PDD arm.

The retrospective cohort design of this study may be considered suboptimal when compared against the methodology of randomized controlled trials. Chou warned about the risks of population and selection heterogeneity in their review[9]. However, by applying strict inclusion and exclusion criteria, we aimed to reduce this heterogeneity (e.g., including only newly presenting patients, having strict rules for resection quality, and ensuring adjuvant intravesical therapy). Compared with the randomized controlled trials reported in earlier systematic reviews, our sample size was typically larger, the inclusion criteria were stricter, and the follow-up period was the longest[5-9]. Additionally, we applied propensity regression adjustment in order to correct for possible confounding covariates. We did not perform propensity sore case matching as this is not superior to using propensity scores in regression analysis as suggested by D'agostino et al [15]. Therefore, we believe this study supplements the existing pool of data from randomized controlled trials and gives a valid representation of how PDD for TUR affects outcomes in a real-world setting. We believe that studies like ours are needed, especially because meta-analyses were inconclusive and we expect that no new prospective studies will be added to the literature.

Nevertheless, our results conflict with those of Gallagher[11], and we have been unable to explain this discrepancy based on differences in patient characteristics or treatments. The baseline number of T1 tumors in the PDD group was smaller in our study (6.6\%) than in the study by Gallagher (23.4\%), but this difference was nullified by stratification into EORTC risk groups. Indeed, distributions were consistent among the low-, intermediate-, and high-risk groups for both populations (our study: 29.7\%, 37\%, and 33.3\%, respectively; Gallagher: $29.7 \%, 40.6 \%$, and $29.7 \%$, respectively). Finally, the use of adjuvant intravesical therapy could only be compared for BCG use, and there was no significant difference between the PDD group (20.3\%) and WLC group (16.5\%).

\section{Conclusions}

In conclusion, the use of PDD TUR for suspected NMIBC did not reduce the RR compared to standard WLC in our retrospective analysis of real-world data. The role of PDD in reducing disease recurrence when used in the management of NMIBC remains unclear given the conflicting research data from both randomized controlled 
trials and observational studies. To address the limitations in this research, further research is warranted in real-world settings

\section{Declarations}

\section{Authors' contributions:}

FJSH: Protocol/project development; Data collection and management; Data analysis; Manuscript writing.

MHB: Data analysis; Manuscript editing.

ECC: Protocol/project development; Manuscript editing.

MGS: Protocol/project development; Manuscript editing.

\section{Competing interests:}

The authors declare no competing interests.

\section{Data availability:}

The datasets generated during and analysed during the study are available from the corresponding author on reasonable request.

\section{Ethics declaration:}

Ethical approval and informed consent collection was waived by the local Ethics Committee of the Isala Hospital in Zwolle, the Netherlands, in view of the retrospective nature of the study and all the procedures being performed were part of routine care.

\section{References}

1. Babjuk, M. et al. European Association of Urology Guidelines on Non-muscle-invasive Bladder Cancer (TaT1 and Carcinoma In Situ) - 2019 Update. Eur. Urol. 76, 639-657 (2019).

2. Sylvester, R. J. et al. Predicting recurrence and progression in individual patients with stage Ta T1 bladder cancer using EORTC risk tables: A combined analysis of 2596 patients from seven EORTC trials. Eur. Urol. 49, 466-475 (2006).

3. Herr, H. W. \& Donat, S. M. Reduced bladder tumour recurrence rate associated with narrow-band imaging surveillance cystoscopy. BJU Int. 107, 396-398 (2011).

4. Jocham, D. et al. Improved detection and treatment of bladder cancer using hexaminolevulinate imaging: A prospective, phase III multicenter study. J. Urol. 174, 862-866 (2005). 
5. di Stasi, S. M. et al. Hexaminolevulinate hydrochloride in the detection of nonmuscle invasive cancer of the bladder. Ther. Adv. Urol. 7, 339-350 (2015).

6. Burger, M. et al. Photodynamic diagnosis of non-muscle-invasive bladder cancer with hexaminolevulinate cystoscopy: A meta-analysis of detection and recurrence based on raw data. Eur. Urol. 64, 846-854 (2013).

7. Shen, P. et al. Effects of fluorescent light-guided transurethral resection on non-muscle-invasive bladder cancer: a systematic review and meta-analysis. BJU Int. 110, E209-E215 (2012).

8. Yuan, H. et al. Therapeutic Outcome of Fluorescence Cystoscopy Guided Transurethral Resection in Patients with Non-Muscle Invasive Bladder Cancer: A Meta-Analysis of Randomized Controlled Trials. PLoS One 8, 1-8 (2013).

9. Chou, R. et al. Review Article Comparative Effectiveness of Fluorescent Versus White Light Cystoscopy for Initial Diagnosis or Surveillance of Bladder Cancer on Clinical Outcomes: Systematic Review. J. Urol. 197, 548-558 (2017).

10. Mariappan, P. et al. Real-life Experience: Early Recurrence with Hexvix Photodynamic Diagnosis-assisted Transurethral Resection of Bladder Tumour vs Good-quality White Light TURBT in New Non-muscleinvasive Bladder Cancer. Urology 86, 327-331 (2015).

11. Gallagher, K. M. et al. 'Real-life experience': recurrence rate at 3 years with Hexvix®photodynamic diagnosis-assisted TURBT compared with good quality white light TURBT in new NMIBC-a prospective controlled study. World J. Urol. 35, 1871-1877 (2017).

12. Babjuk, M. et al. EAU Guidelines on Non-Muscle-invasive Urothelial Carcinoma of the Bladder: Update 2016. Eur. Urol. 71, 447-461 (2017).

13. Ipsen Limited. Guideline on Summary of Product Characteristics Hexvix. Electronic Medicines Compendium 1 https://www.medicines.org.uk/emc/product/4313/smpc (2004).

14. O’Brien, T. et al. Prospective randomized trial of hexylaminolevulinate photodynamic-assisted transurethral resection of bladder tumour (TURBT) plus single-shot intravesical mitomycin $\mathrm{C}$ vs conventional white-light TURBT plus mitomycin C in newly presenting non-muscle-invasi. BJU Int. 112, 1096-1104 (2013).

15. D’Agostino, R. B. Propensity score methods for bias reduction in the comparison of a treatment to a nonrandomized control group. Stat. Med. 17, 2265-2281 (1998).

\section{Figures}




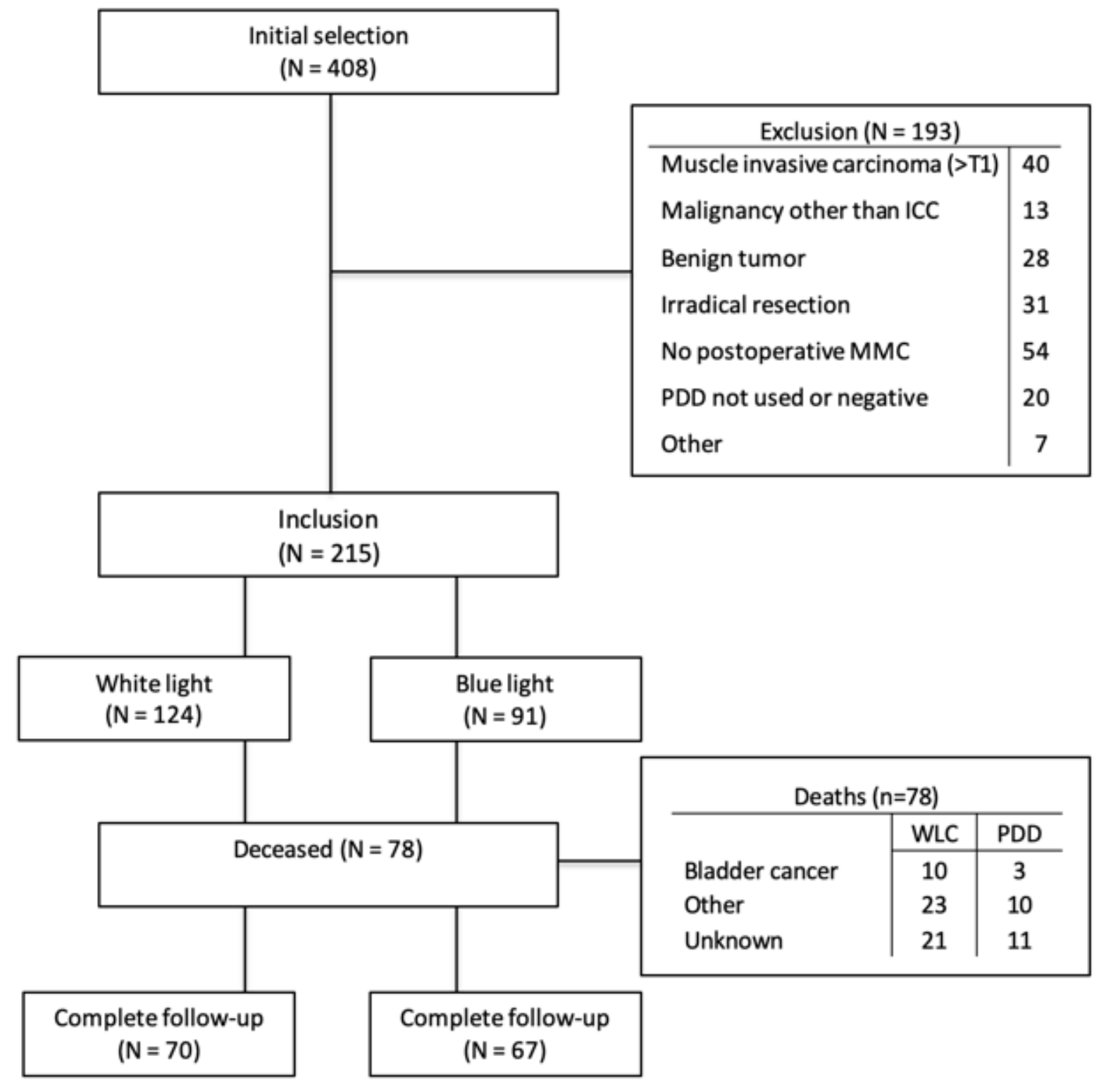

Figure 1

Inclusion procedure flow-chart. Abbreviations: CIS, carcinoma in situ; EORTC, European Organization for Research and Treatment of Cancer; MMC, mitomycin C; PDD, photodynamic diagnosis; UCC, urothelial cell carcinoma; WLC, white light cystoscopy. 


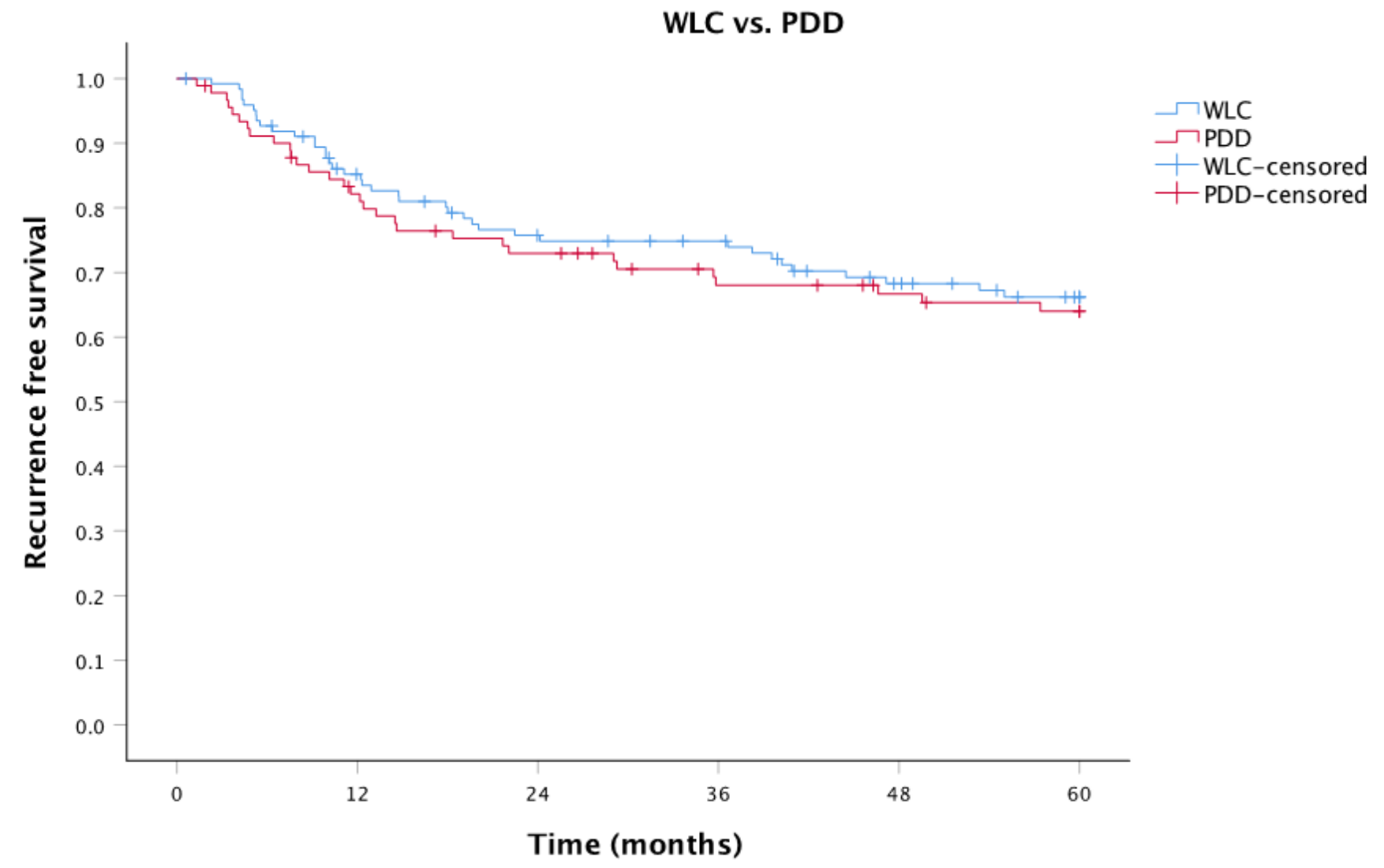

\section{Figure 2}

Kaplan-Meier curve depicting recurrence-free survival by treatment with white light or photodynamic diagnosis. Censoring occurred after loss to follow-up or upon death. Abbreviations: PDD, photodynamic diagnosis; WLC, white light cystoscopy. 